\title{
Immunostimulation and Coagulopathy in COVID-19 Compared to Patients With H1N1 Pneumonia or Bacterial Sepsis
}

\author{
DIMITRIOS-DOROTHEOS PAPADAKIS ${ }^{1}$, MARIANNA POLITOU ${ }^{2}$, MARIA KOMPOTI ${ }^{3}$, \\ DIMITRIOS VAGIONAS ${ }^{1}$, EIRINI KOSTAKOU ${ }^{1}$, DANAI THEODOULOU ${ }^{1}$, \\ EVANGELOS KANIARIS ${ }^{1}$, NIKOLETTA ROVINA ${ }^{1}$, GEORGE PANAYIOTAKOPOULOS ${ }^{4}$, \\ STAVROS DIMOPOULOS $^{5}$, ANTONIA KOUTSOUKOU ${ }^{1}$ and IOANNIS VASILEIADIS ${ }^{5}$ \\ ${ }^{1}$ Intensive Care Unit, $1^{\text {st }}$ Department of Respiratory Medicine, \\ National and Kapodistrian University of Athens, Sotiria Hospital, Athens, Greece; \\ ${ }^{2}$ Haematology Laboratory-Blood Bank, National and Kapodistrian University of Athens, \\ Aretaieion Hospital, Athens, Greece; \\ ${ }^{3}$ Thriassio General Hospital of Eleusis, Eleusis, Greece; \\ ${ }^{4}$ Pharmacology Laboratory, Medical School, Patras University, Rio, Greece; \\ ${ }^{5} 1^{\text {st }}$ Critical Care Department, National and Kapodistrian University of Athens, \\ Evangelismos Hospital, Athens, Greece
}

\begin{abstract}
Background/Aim: Multiple reports from all over the world link COVID-19 with endothelial/coagulation disorders as well as a dysregulated immune response. This study tested the hypothesis that immunostimulation will be greater in COVID-19 patients than in patients with HINI infection or bacterial sepsis. Also, whether an increase in immune stimulation will be accompanied by a more severely affected endothelium/coagulation system was examined. Patients and Methods: Twenty-three septic patients, admitted in the Intensive Care Unit (ICU), were enrolled (9 with SARS-CoV-2, 5 with HINI pneumonia, 9 with bacterial sepsis). Myeloperoxidase (MPO) activity along with certain endothelial/coagulation factors were assessed on admission (time point 1) and at either improvement or deterioration (time point 2). Results: MPO levels were significantly higher in COVID-19 patients compared to both other groups. Furthermore, in patients with COVID-19, vWF levels did not differ significantly, fVIII levels were lower while ADAMTS13 activity was higher compared to patients with H1N1
\end{abstract}

This article is freely accessible online.

Correspondence to: Ioannis Vasileiadis, MD, Ph.D., Associate Professor of Internal Medicine and Critical Care, National and Kapodistrian University of Athens, School of Medicine, $1^{\text {st }}$ Critical Care Department, Evangelismos Hospital, Ipsilantou 45-47, 10676, Athens, Greece. Tel: +30 2132043385, Mob: +30 6977644866, e-mail: ioannisvmed@yahoo.gr, ivasileiadis@med.uoa.gr

Key Words: Innate immunity, SARS-CoV-2, coagulopathy in COVID-19, ADAMTS-13 pneumonia and bacterial sepsis (a trend in the latter). Conclusion: Increased immunostimulation was noted in COVID-19 patients compared to other septic patients; however, this was not accompanied by greater disturbance of the clotting system and/or more severe endothelial injury.

Since the novel coronavirus disease (COVID-19) epidemic broke out, knowledge of its underlying pathophysiology has progressively risen. Among other peculiar features, immune response dysregulation has been reported (1), e.g., high levels of neutrophil extracellular traps (NETs) may contribute to cytokine release and respiratory failure in these patients (2). Moreover, there is increased interest regarding the possible role of impaired microcirculation in the pathogenesis of severe acute respiratory syndromecoronavirus-2 (SARS-CoV-2) disease. Various reports from all over the world link COVID-19 with endothelial dysfunction (3) and coagulation abnormalities (4).

It has been already recognized that inflammation induced during sepsis can trigger thrombosis. This phenomenon, initiated by innate immunity activation, is referred to as immunothrombosis (5). Thrombi formation in microvessels is likely to play an important role in the body's defense against pathogenic invaders. However, if uncontrolled, it can lead organ failure, endangering the life of the host.

A specific immune effector that has drawn interest, as it has been associated with microthrombi formation, is the neutrophilic enzyme myeloperoxidase (MPO). MPO is a long-known lysosomal protein, stored in the azurophilic granules of neutrophils and released into the extracellular space during degranulation, as a response to infection or 
other inflammatory stimuli. MPO imparts its antimicrobial properties by converting hydrogen peroxide into hypochlorous acid (6). Aside from its antimicrobial properties, the aforementioned enzyme seems to have prothrombotic activity as it inhibits von Willebrand factor (vWF) cleavage by ADAMTS-13 (a disintegrin and metalloproteinase with a thrombospondin type 1 motif, member 13) (7). MPO also participates in the neutrophil extracellular traps (NETs) formation (8). MPO-DNA levels have already been measured in COVID-19 patients and were found to be elevated in a study that correlated SARS-CoV-2 infection with NETs formation (2).

Reports regarding specific differences of clotting and immune system disorders between critically ill, COVID-19 patients and patients with sepsis of other etiology are scant. We hypothesized that immunostimulation will be greater in COVID-19 patients than in patients with H1N1 infection or bacterial sepsis. Also, that the greater immune stimulation will be accompanied by a more severely affected endothelium/coagulation system.

The aim of this study was to investigate the immune system activation, by measuring MPO levels, along with hemostatic and endothelium related factors, in critically ill COVID-19 patients, compared to patients with H1N1 pneumonia or bacterial sepsis, hospitalized to the Intensive Care Unit (ICU).

\section{Patients and Methods}

Patient cohort. This was a prospective, observational study that took place in a 10-bed adult ICU during a 4-month period, between January and April 2020. The study was conducted according to the guidelines of the Declaration of Helsinki and approved by the Institutional Ethics Committee of Sotiria Hospital, Athens, Greece (Institutional Ethics Committee approval number: 8854/18-9-2020). Patients themselves or their next of kin provided informed consent. Patients with bacterial sepsis, H1N1 pneumonia or SARS-CoV-2 infection who met the sepsis criteria (9) at the time of ICU admission were consecutively enrolled in the study. As it becomes clear, the blood sampling took place during the first wave of the pandemic and as a result none of the patients were vaccinated with a SARS-CoV-2 vaccine.

Upon admission (baseline or time point 1), blood samples were drawn and analyzed for routine laboratory parameters and each enrolled patient's Acute Physiology and Chronic Health Evaluation (APACHE) II (10) and Sequential Organ Failure Assessment (SOFA) (11) scores were calculated. Serum and plasma samples were stored at $-80^{\circ} \mathrm{C}$ in order to estimate ADAMTS-13 activity, vWF, factor VIII (fVIII), antithrombin III (ATIII), protein C, protein S, myeloperoxidase (MPO) and anticardiolipin antibodies (ACA). vWF/ADAMTS-13 ratio was also calculated. Assessment of the same parameters and SOFA score calculation was repeated when a significant change in the patients' clinical condition was noted, either remission or prominent deterioration (time point 2), defined as elsewhere (12). Particularly, sepsis remission was defined as: i) Improvement of the systemic inflammatory response syndrome (SIRS) indices, mainly the temperature $\left(\mathrm{T}, 36^{\circ} \mathrm{C} \leq \mathrm{T} \leq 38^{\circ} \mathrm{C}\right)$ and the white blood cell count (WBC), 4,000/ $\mu \mathrm{l} \leq \mathrm{WBC} \leq 12,000 / \mu 1$ and $\leq 10 \%$ immature forms, ii) Hemodynamic stability for at least $24 \mathrm{~h}$ (systolic blood pressure $\geq 90 \mathrm{~mm} \mathrm{Hg}$ and mean blood pressure $\geq 65 \mathrm{~mm} \mathrm{Hg}$ ), with no need for vasoactive agents, and lactate concentration $<2 \mathrm{mEq} / \mathrm{l}$, and iii) Recovery of organ failures attributed to sepsis, with restoration of organ dysfunction variables and a SOFA score reduction $\geq 2$ compared to the SOFA score at time point 1 . Although SIRS criteria are no longer included in the current definition of sepsis, we used these markers that are commonly used by ICU physicians for the clinical assessment of the progress of sepsis. If a patient was discharged from the ICU, being considered cured by the ICU physicians, we recorded the parameter values on the day of ICU exit. Also, sepsis deterioration was defined as: a) Deterioration of hemodynamic instability, with a minimum score of three points in the hemodynamic category, as settled on the SOFA severity scale, and increase of lactate levels $>1 \mathrm{mEq} / \mathrm{l}$ compared to time point 1 , up to a concentration of $\geq 2 \mathrm{mEq} / \mathrm{l}$ or increased needs for vasoactive substances (norepinephrine increase $>0.1 \mu \mathrm{g} / \mathrm{kg} / \mathrm{min}$, addition of epinephrine), with the exception of hemorrhagic shock, cardiogenic shock due to acute cardiac episode or obstructive shock, and b) Deterioration of organ failure due to the septic syndrome, with a subsequent SOFA score $\geq 1$ increase compared to the SOFA score at time point 1 (12).

The diagnosis of both SARS-CoV-2 and H1N1 infections were made in the Hellenic Pasteur Institute by applying in-house realtime polymerase chain reaction (RT-PCR) on bronchial secretions. For bacterial infections, blood, urine, and bronchial secretion cultures were utilized.

Biochemical indices. Serum Iron was measured by the Medilyzer ${ }^{\circledR}$ Bx biochemical analyzer (MEDICON HELLAS S.A., Athens, Greece) using the chemiluminescence method. Serum Ferritin values were acquired by using the TheAlinityi Ferritin Immunoassay (Abbott Laboratories, Chicago, IL, USA) which is a chemiluminescent microparticle immunoassay (CMIA). d-dimers, ATIII, Prot $C$, free Prot S, VWF activity, fVIII: d-dimers [INNOVANCE D-Dimer, a particle-enhanced immunoturbidimetric assay for the quantitative determination of cross-linked fibrin degradation products (D-dimers)], ATIII [Berichrom ${ }^{\circledR}$ AT III (A)], fVIII (Coagulation Factor VIII Deficient plasma), Protein $\mathrm{C}$ (ProC $\left.{ }^{\circledR} \mathrm{Ac} \mathrm{R}^{* *}\right)$, free Protein $\mathrm{S}$ (INNOVANCE Free PS Ag2), and VWF activity (INNOVANCE VWF Ac**) were determined by the Siemens coagulation analyzer BCS/BCS XP System (Siemens Medical Solutions USA, Inc., Malvern, PA, USA), employing the chromogenic method. ADAMTS13 activity was measured by fluorescence resonance energy transfer (FRET) technology (ADAMTS-13Activity Assay, ATS-13, GTI Diagnostics, Waukesha, WI, USA) with a Fluoroskan Ascent fluorometer (Thermo Scientific, Helsinki, Finland). Myeloperoxidase values were determined by Quantikine ${ }^{\circledR}$ Human MPO Immunoassay, employing ELISA (R\&D Systems, Minneapolis, MN, USA). Anticardiolipin antibodies were measured by QUANTA Lite ${ }^{\circledR}$ ACA Screen III Assay, employing ELISA (R\&D Systems).

Statistical analysis. Categorical variables were analyzed with the Fisher's exact test. Normality of continuous variables was assessed with Kolmogorov-Smirnov test. Continuous variables (baseline and time point 2) were compared with a non-parametrical method (Wilcoxon's test), while for comparisons of continuous variables on time point 2 according to outcome (deterioration/improvement) Mann-Whitney test was implemented. Repeatedly measured variables 
Papadakis et al: Immunostimulation and Coagulopathy in COVID Sepsis

Table I. Comparison of demographics and of clinical and laboratory parameters upon intensive care unit (ICU) admission among the 3 groups of septic patients.

\begin{tabular}{|c|c|c|c|c|c|}
\hline Variable & $\begin{array}{l}\text { Total } \\
(n=23)\end{array}$ & $\begin{array}{l}\text { COVID-19 } \\
\quad(\mathrm{n}=9)\end{array}$ & $\begin{array}{l}\text { H1N1 pneumonia } \\
(\mathrm{n}=5)\end{array}$ & $\begin{array}{l}\text { Bacterial sepsis } \\
\qquad(\mathrm{n}=9)\end{array}$ & $p$-Value \\
\hline Male gender (\%) & $16(69.6)$ & $6(66.7)$ & $4(80)$ & $6(66.7)$ & 0.848 \\
\hline Age (years) & $65.1 \pm 12.5$ & $61.9 \pm 13.4$ & $67 \pm 11.7$ & $67.3 \pm 12.9$ & 0.359 \\
\hline APACHE II & $19.6 \pm 8.7$ & $14.3 \pm 5.2$ & $25.8 \pm 11.5$ & $21.3 \pm 7.7$ & 0.069 \\
\hline SOFA & $7.5 \pm 2.0$ & $6.6 \pm 2.1$ & $7.2 \pm 1.5$ & $8.6 \pm 1.7$ & 0.100 \\
\hline ICU LOS (days) & $15.9 \pm 11.1$ & $15.4 \pm 5.6$ & $20.2 \pm 16.1$ & $13.9 \pm 12.2$ & 0.440 \\
\hline Deterioration (\%) & $6(26.1)$ & $2(22.2)$ & $0(0)$ & $4(44.4)$ & 0.182 \\
\hline Time interval (days) & $11.5 \pm 9.7$ & $11.7 \pm 6.2$ & $15.2 \pm 13.6$ & $9.3 \pm 10.7$ & 0.396 \\
\hline Ferritin $(\mathrm{ng} / \mathrm{ml})$ & $954.3 \pm 600$ & $1,333.7 \pm 429.2$ & $607.3 \pm 513.8$ & $767.6 \pm 630.4$ & 0.047 \\
\hline d-dimers $(\mathrm{mg} / \mathrm{l})^{\mathrm{a}}$ & $1.77(0.98-3.94)$ & $1.77(0.78-3.87)$ & $1.06(0.96-18.73)$ & $2.89(1.26-6.8)$ & 0.400 \\
\hline Fibrinogen (mg/dl) & $624 \pm 193$ & $727 \pm 177$ & $420 \pm 78$ & $586 \pm 176$ & 0.035 \\
\hline $\mathrm{WBC}(/ \mu \mathrm{l})^{\mathrm{a}}$ & $12,230(8,810-15,440)$ & $11,320(8,055-12,005)$ & $16,820(9,125-17,950)$ & $13,950(9,895-16,155)$ & 0.079 \\
\hline Neutrophils $(/ \mu \mathrm{l})$ & $9,937 \pm 4,030$ & $7,678 \pm 3,141$ & $11,076 \pm 5,009$ & $11,562 \pm 3,567$ & 0.082 \\
\hline $\operatorname{PLT}\left(\times 10^{3}\right)(/ \mu \mathrm{l})$ & $254.5 \pm 128.5$ & $231.8 \pm 88.0$ & $388.8 \pm 146.6$ & $202.6 \pm 109.6$ & 0.05 \\
\hline Serum iron $(\mathrm{mg} / \mathrm{dl})$ & $40.1 \pm 26.7$ & $40.6 \pm 30.9$ & $43.2 \pm 30.8$ & $38.0 \pm 22.8$ & 0.859 \\
\hline $\mathrm{CRP}(\mathrm{mg} / \mathrm{dl})$ & $16.1 \pm 9.4$ & $16.8 \pm 7.7$ & $11.9 \pm 9.4$ & $17.8 \pm 11.3$ & 0.527 \\
\hline PCT $(\mathrm{ng} / \mathrm{ml})^{\mathrm{a}}$ & $0.7(0.27-1.95)$ & $0.54(0.27-0.84)$ & $0.31(0.24-5.34)$ & $1.79(0.43-4.3)$ & 0.233 \\
\hline $\operatorname{ATIII}(\%)$ & $80.5 \pm 33.3$ & $77.3 \pm 35.9$ & $86.7 \pm 22.0$ & $80.2 \pm 38.5$ & 0.820 \\
\hline Protein C $(\%)$ & $70.2 \pm 56.8$ & $53.9 \pm 15.0$ & $59.9 \pm 15.9$ & $92.3 \pm 87.2$ & 0.267 \\
\hline Protein S (\%) & $92.9 \pm 25.5$ & $86.9 \pm 26.8$ & $97.4 \pm 14.7$ & $96.3 \pm 29.9$ & 0.713 \\
\hline $\mathrm{vWF}(\mathrm{IU} / \mathrm{ml})$ & $408.9 \pm 145.3$ & $369.5 \pm 147.1$ & $412.7 \pm 141.4$ & $529.4 \pm 154.0$ & 0.644 \\
\hline fVIII $(\%)$ & $143.0 \pm 44.9$ & $115.1 \pm 21.5$ & $151.8 \pm 55.4$ & $166.1 \pm 45.0$ & 0.047 \\
\hline ADAMTS-13 $(\%)$ & $51.5 \pm 36.8$ & $72.0 \pm 32.7$ & $22.3 \pm 15.1$ & $47.2 \pm 37.9$ & 0.020 \\
\hline vWF/ADAMTS-13 ratio & $28.2 \pm 36.7$ & $14.2 \pm 27.3$ & $39.2 \pm 32.4$ & $36.2 \pm 45.5$ & 0.084 \\
\hline MPO (ng/ml) & $469.6 \pm 404.3$ & $743.9 \pm 357.5$ & $267.5 \pm 319.3$ & $307.6 \pm 362.0$ & 0.017 \\
\hline $\mathrm{ACA}(\%)$ & $10(43.5)$ & $6(66.7)$ & $2(40)$ & $2(22.2)$ & 0.161 \\
\hline
\end{tabular}

aMedian (interquartile range). Post hoc analysis for multiple comparisons was performed with Dunn's test. APACHE: Acute physiology and chronic health evaluation; SOFA: sequential organ failure assessment; ICU: intensive care unit; LOS; length of stay; WBC: white blood cell count; PLT: platelets; CRP: C-reactive protein; PCT: procalcitonin; ATIII: antithrombin III; vWF: von Willebrand factor; fVIII: factor VIII; ADAMTS-13: a disintegrin and metalloprotease with thrombospondin type 1 motifs 13; MPO: myeloperoxidase; ACA: anticardiolipin antibodies.

were entered in linear regression models fitted with generalized estimating equations (GEE), an extension of the generalized linear model that accounts for within-subject correlation. Time was entered as a two-level ordinal variable (level 1: baseline, level 2: time-point 2 ) and was treated as a within-subject correlation variable. GEE linear models for various response variables (MPO, ADAMTS-13, vWF etc.) were fitted and tested. Furthermore, more complex models with various sets of explanatory variables were tested. In all GEE models an unstructured correlation structure was used and the quasi likelihood information criterion (QIC) was used for model selection. Discriminating ability of MPO for COVID-19 was assessed with receiver operating characteristic (ROC) analysis. Data analysis was performed with SPSS 17.0 (IBM Corporation, NY, USA, 2008). For all analyses, alpha was set at 0.05 (two-tailed).

\section{Results}

We studied 16 male and seven female patients, with a mean age $( \pm$ SD) of $65.1 \pm 12.5$ years. All patients met the Sepsis-3 criteria. All patients were intubated and mechanically ventilated upon their ICU admission. Septics with respiratory infection (most of the cohort) had a $\mathrm{PaO}_{2} / \mathrm{FiO}_{2}$ ratio less than 150 .
Mean ICU length of stay (ICU-LOS) was $15.9 \pm 11.1$ days. Mean APACHE II score on admission was 19.6 \pm 8.7 . Five patients died in the ICU $(21.7 \%)$. Nine patients were diagnosed with COVID-19 infection, five patients with H1N1 pneumonia and nine patients had bacterial sepsis. Among the COVID-19 patients, 2 suffered from COPD, 1 from asthma, 3 from arterial hypertension and 2 from diabetes. Four of them were active smokers. None of the patients had heart failure of NYHA stage III or IV. In total, in the present study, 9 patients had COPD, 5 had type II diabetes mellitus, 9 had arterial hypertension, 2 had chronic heart failure, and 1 had stage 3 chronic kidney disease. Eight patients were active smokers, and 6 had a history of dyslipidemia. Among the deceased patients, 4 had bacterial Sepsis and 1 was a COVID-19 patient. None of the Influenza patients died during their stay in the ICU.

Table I summarizes clinical and laboratory results of our patient population on admission. Differences between the baseline values (upon ICU admission, time point 1) and at either improvement or deterioration (time point 2) are 
Table II. Endothelial and coagulation variables in septic patients upon ICU admission and at either sepsis remission (group A) or deterioration (group B).

\begin{tabular}{|c|c|c|c|c|c|c|}
\hline & \multicolumn{2}{|c|}{ Group A (n=17) } & \multirow[t]{2}{*}{$p$-Value } & \multicolumn{2}{|c|}{ Group B $(n=6)$} & \multirow[t]{2}{*}{$p$-Value } \\
\hline & $\begin{array}{c}\text { Admission } \\
\text { (Time point } 1 \text { ) }\end{array}$ & $\begin{array}{l}\text { Improvement } \\
\text { (Time point 2) }\end{array}$ & & $\begin{array}{c}\text { Admission } \\
\text { (Time point 1) }\end{array}$ & $\begin{array}{l}\text { Deterioration } \\
\text { (Time point 2) }\end{array}$ & \\
\hline SOFA & $7.1 \pm 1.9$ & $2.6 \pm 1.7$ & $<0.001$ & $8.5 \pm 1.9$ & $10.3 \pm 1.6$ & 0.026 \\
\hline ATIII (\%)* & $89.7 \pm 29.3$ & $111.3 \pm 14.1$ & 0.001 & $54.2 \pm 31.9$ & $59.9 \pm 35.0$ & 0.463 \\
\hline Protein C (\%) & $61.3 \pm 11.0$ & $63.4 \pm 17.0$ & 0.723 & $56(27.3-147.5)$ & $31.2(14.6-54.4)$ & 0.075 \\
\hline Protein S (\%) & $98.9 \pm 19.4$ & $122.7 \pm 20.4$ & 0.003 & $75.9 \pm 34.4$ & $67.4 \pm 31.4$ & 0.138 \\
\hline vWF (IU/ml) & $413.4 \pm 134.5$ & $423.4 \pm 139.7$ & 0.833 & $401.9(206.1-599)$ & $599(592.4-599)$ & 0.068 \\
\hline fVIII $(\%)$ & $135.9 \pm 43.0$ & $215.1 \pm 88.7$ & 0.001 & $163.2 \pm 48.0$ & $264.7 \pm 58.2$ & 0.028 \\
\hline ADAMTS-13 $(\%)$ & $50.4 \pm 39.8$ & $56.0 \pm 36.9$ & 0.569 & $54.5 \pm 29.5$ & $38.8 \pm 22.9$ & 0.043 \\
\hline vWF/ADAMTS-13 ratio & $29.5 \pm 34.2$ & $22.5 \pm 33.5$ & 0.492 & $6.2(3.2-37.6)$ & $13.5(10.2-54.7)$ & 0.043 \\
\hline MPO (ng/ml) & $451.7 \pm 408.5$ & $508.1 \pm 422.4$ & 0.906 & $551.4(63.9-913.1)$ & $238.2(86.8-567.0)$ & 0.345 \\
\hline
\end{tabular}

*ATIII at baseline was lower in septics who deteriorated compared to those who improved $(p=0.02)$. ICU: Intensive care unit; SOFA: sequential organ failure assessment; ATIII: Antithrombin III; vWF: von Willebrand factor; fVIII: factor VIII; ADAMTS-13: a disintegrin and metalloprotease with thrombospondin type 1 motif 13; MPO: myeloperoxidase.

Table III. Linear regression models for myeloperoxidase.

\begin{tabular}{lccc}
\hline & & Myeloperoxidase (adjusted for APACHE II) \\
\hline Infection type & & $(-708.6)-(-328.1)$ & $<0.001$ \\
Bacterial sepsis & -518.4 & $(-671.5)-(-159.5)$ & 0.001 \\
H1N1 & -415.5 & Myeloperoxidase (adjusted for vWF) \\
Covid-19 (ref) & 0 & & $(-686.5)-(-360.9)$ \\
\hline & & $(-634.9)-(-239.1)$ & $<0.001$ \\
Infection type & -523.7 & $(-5.7)-(-0.7)$ & $<0.001$ \\
Bacterial sepsis & -447.0 & 0 & 0.013 \\
H1N1 & 0 & & \\
ADAMTS-13 (per percentile increase) & -3.2 & & \\
\hline
\end{tabular}

ADAMTS-13: A disintegrin and metalloprotease with thrombospondin type 1 motif 13; APACHE: acute physiology and chronic health evaluation; vWF: von Willebrand factor.

shown in Table II. Linear regression models for Myeloperoxidase are displayed in Table III, while MPO levels identified in each type of infection studied are presented in Figure 1.

Most importantly, MPO was significantly higher in COVID19 patients compared to both other groups (Table II, Figure 1), and this strong independent association persisted in all fitted models, after adjustment for various potential confounders (disease severity, organ failure, patient outcome). MPO displayed good discriminating ability for COVID-19, with area under curve (AUC) at baseline 0.86 (95\% confidence interval $=0.66-1, p=0.005)$. A $258 \mathrm{ng} / \mathrm{ml}$ cutoff at baseline displayed $89 \%$ sensitivity and $72 \%$ specificity for COVID-19 detection. A $756 \mathrm{ng} / \mathrm{ml}$ cutoff at baseline displayed 86\% specificity for COVID-19 detection. At time point 2, regardless of patient status (improvement/deterioration), MPO discriminating ability persisted (AUC $=0.83,95 \%$ confidence interval $=0.62-1.0, p=0.01)$.

\section{Discussion}

The main finding of the study was the consistently elevated MPO levels in patients with COVID-19 compared to critically ill patients with H1N1 pneumonia or bacterial sepsis, indicating an increased inflammatory response in the context of innate immunity activation. 


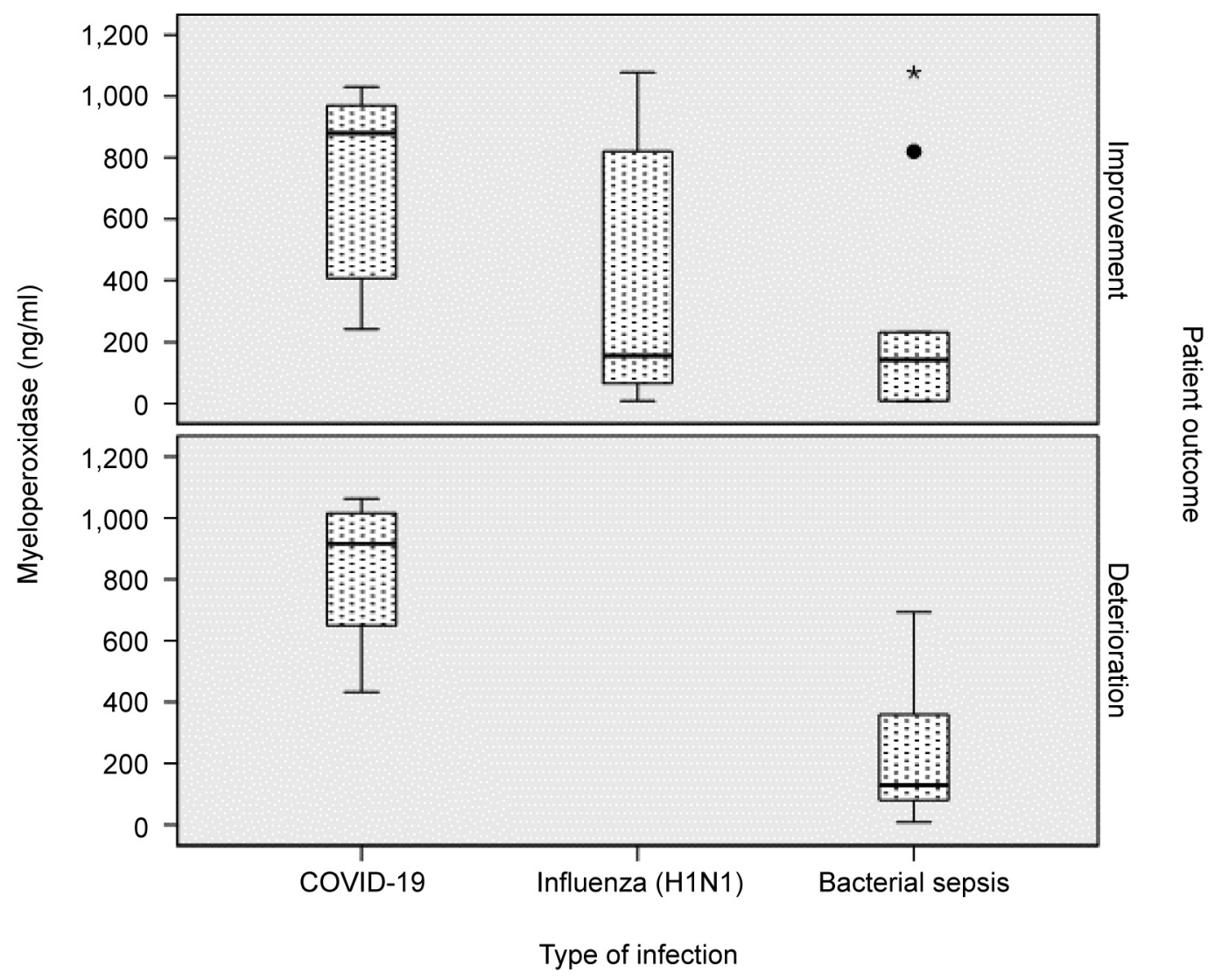

Figure 1. Myeloperoxidase levels in each type of infection.

Levels of MPO-DNA along with cell-free DNA and citrullinated histone $\mathrm{H} 3$ (Cit-H3), all markers of NETosis, measured in COVID-19 patients, have also been found to be elevated compared to healthy controls. Although NETs exert infection-containing action, upon dysregulation, become sentinels of microvascular thrombosis and inflammation, as observed -among others- in lung tissue of acute respiratory distress syndrome patients. The clinical relevance of these observations is magnified by the association of cell-free DNA with acute-phase reactants, the correlation of MPODNA with absolute neutrophil count and cell-free DNA, and Cit-H3 correlation with platelet levels. Evidently, elevation of both MPO-DNA and cell-free DNA has also been identified among mechanically-ventilated patients as compared to hospitalized patients breathing room air, further highlighting the importance of a dysregulated MPO system in the progression of COVID-19 (2).

MPO was negatively associated with ADAMTS-13 (Table II), in accordance with studies showing that ADAMTS-13 activity is reduced when exposed to hypochlorous acid produced by an MPO system (13). At the same time, ADAMTS-13 activity was greater in COVID-19 patients, who had higher MPO levels compared to the other patient groups. Although this may seem contradictory at first glance, it should be noted that the latter refers to the recorded absolute values of these biological molecules, while the former to their concurrent change during the two time-point period. There is, therefore, no question of a contradiction between the recorded findings. No significant effect of MPO was noted on other coagulation variables. Regarding the MPO levels and their variation at the different time points in the groups of patients studied, pertinent remarks are provided.

In the entire patient cohort, MPO levels increased when septic condition improved and decreased when septic condition worsened (although the differences were not statistically significant); that is reminding that MPO system plays a very important role in the microbicidal activity of phagocytes, decisively contributing to the effective management of the infectious invaders, i.e., MPO is primarily considered a friend of the human organism, not a foe (14). On the other hand, MPO levels in COVID-19 patients remained elevated at both, improvement, and deterioration. Indeed, persistently high inflammatory activity may be observed for a long period of time after an acute 
infectious disease; thus, in patients treated for an infectious disease (i.e., community acquired pneumonia), certain inflammatory markers may remain elevated even after hospital discharge, being associated with increased mortality, even a year later (15). Particularly relating the MPO system, a dual role of MPO has been proposed in immune response; thus, apart from its apparently beneficial role, MPO may also have harmful effects, disturbing tissue homeostasis and being involved in the pathological process of tissue damage and disease progression (16).

The above observations actually point to the fact that infection by a pathogenic microorganism and the body's response to it is a complex phenomenon, which can take different clinical forms, from a cataclysmic inflammatory response to an absolute immune paralysis. The effect of any individual biomolecule activity on outcome cannot be simplistically and unambiguously determined/predicted. Even more so, since the onset of this disease is still only recent, and its specific behavior, evolution and possible longterm effects are under constant scrutiny.

Finally, in patients with COVID-19, vWF levels did not differ significantly, fVIII levels were lower while ADAMTS13 activity was higher compared to patients with H1N1 pneumonia and bacterial sepsis (a trend in the latter). Thus, coagulation/endothelial disorders appeared to be less pronounced in COVID-19 patients. To note, COVID-19 patients did not have clinical signs of microangiopathy.

In the whole cohort, vWF/ADAMTS-13 ratio increased with disease severity, as previously shown (17). High ferritin and fibrinogen levels as acute-phase reactants were also confirmed in our cohort of COVID-19 patients (18).

As shown in Table II, at deterioration, the observed hemostatic factor changes were rather expected with patients that deteriorated having higher vWF/ADAMTS13 ratio. Interestingly, at improvement, (a point when patients were considered able to exit ICU) factors associated with endothelial injury/activation (e.g., fVIII, vWF) did not improve, in accordance with previous studies (12). Hence, endothelial damage appears to persist for some time following the septic stimulus (19); in another study, secondary activation of oxidase system in circulating phagocytes, long after the clinical syndrome has resolved, was observed (20).

Limitations. A limitation of this study is the small number of patients enrolled. Nevertheless, the main finding of our study (MPO difference), in the cohort of the consecutive patients studied, is robust, persisting in all fitted models. Moreover, as the disturbance of certain coagulation/endothelial factors, that were assessed in the same cohort of patients at the same time points, has generally been associated with enhanced immune stimulation, our results do not prevent some conclusions from being drawn: i.e., greater immune stimulation in COVID-19 does not appear to be a sufficient, at least, condition for causing more severe coagulation/endothelial disorders; other factors might possibly play an important role. Nevertheless, further studies are likely to decide whether it is a necessary condition.

\section{Conclusion}

In conclusion, immune system stimulation was enhanced in COVID-19 septic patients compared to patients with $\mathrm{H} 1 \mathrm{~N} 1$ pneumonia or bacterial sepsis; however, this did not result in greater disturbance of the clotting system and/or more severe endothelial injury.

\section{Conflicts of Interest}

The Authors declare no conflicts of interest.

\section{Authors' Contributions}

Conceptualization, Marianna Politou and Ioannis Vasileiadis; Data curation, Dimitrios-Dorotheos Papadakis, Eirini Kostakou, Danai Theodoulou, Evangelos Kaniaris, Nikoletta Rovina, George Panayiotakopoulos and Stavros Dimopoulos; Formal analysis, Maria Kompoti; Writing - original draft, Dimitrios-Dorotheos Papadakis, Dimitrios Vagionas and Ioannis Vasileiadis; Writing - review \& editing, Marianna Politou, Antonia Koutsoukou and Ioannis Vasileiadis.

\section{References}

1 Qin C, Zhou L, Hu Z, Zhang S, Yang S, Tao Y, Xie C, Ma K, Shang K, Wang W and Tian DS: Dysregulation of immune response in patients with Coronavirus 2019 (COVID-19) in Wuhan, China. Clin Infect Dis 71(15): 762-768, 2020. PMID: 32161940. DOI: $10.1093 / \mathrm{cid} / \mathrm{ciaa} 248$

2 Zuo Y, Yalavarthi S, Shi H, Gockman K, Zuo M, Madison JA, Blair C, Weber A, Barnes BJ, Egeblad M, Woods RJ, Kanthi Y and Knight JS: Neutrophil extracellular traps in COVID-19. JCI Insight 5(11): e138999, 2020. PMID: 32329756. DOI: 10.1172/ jci.insight 138999

3 Varga Z, Flammer AJ, Steiger P, Haberecker M, Andermatt R, Zinkernagel AS, Mehra MR, Schuepbach RA, Ruschitzka F and Moch H: Endothelial cell infection and endotheliitis in COVID19. Lancet 395(10234): 1417-1418, 2020. PMID: 32325026. DOI: $10.1016 / \mathrm{S} 0140-6736(20) 30937-5$

4 Fogarty H, Townsend L, Ni Cheallaigh C, Bergin C, MartinLoeches I, Browne P, Bacon CL, Gaule R, Gillett A, Byrne M, Ryan K, O'Connell N, O'Sullivan JM, Conlon N and O'Donnell JS: COVID19 coagulopathy in Caucasian patients. $\mathrm{Br} \mathrm{J}$ Haematol 189(6): 1044-1049, 2020. PMID: 32330308. DOI: 10.1111/bjh.16749

5 Engelmann B and Massberg S: Thrombosis as an intravascular effector of innate immunity. Nat Rev Immunol 13(1): 34-45, 2013. PMID: 23222502. DOI: $10.1038 /$ nri3345

6 Astern JM, Pendergraft WF 3rd, Falk RJ, Jennette JC, Schmaier $\mathrm{AH}$, Mahdi $\mathrm{F}$ and Preston GA: Myeloperoxidase interacts with endothelial cell-surface cytokeratin 1 and modulates bradykinin production by the plasma Kallikrein-Kinin system. Am J Pathol 171(1): 349-360, 2007. PMID: 17591979. DOI: 10.2353/ ajpath.2007.060831 
7 Chen J, Fu X, Wang Y, Ling M, McMullen B, Kulman J, Chung DW and López JA: Oxidative modification of von Willebrand factor by neutrophil oxidants inhibits its cleavage by ADAMTS13. Blood 115(3): 706-712, 2010. PMID: 19812385. DOI: 10.1182/blood-2009-03-213967

8 Papayannopoulos V, Metzler KD, Hakkim A and Zychlinsky A: Neutrophil elastase and myeloperoxidase regulate the formation of neutrophil extracellular traps. J Cell Biol 191(3): 677-691, 2010. PMID: 20974816. DOI: 10.1083/jcb.201006052

9 Singer M, Deutschman CS, Seymour CW, Shankar-Hari M, Annane D, Bauer M, Bellomo R, Bernard GR, Chiche JD, Coopersmith CM, Hotchkiss RS, Levy MM, Marshall JC, Martin GS, Opal SM, Rubenfeld GD, van der Poll T, Vincent JL and Angus DC: The third international consensus definitions for sepsis and septic shock (Sepsis-3). JAMA 315(8): 801-810, 2016. PMID: 26903338. DOI: 10.1001/jama.2016.0287

10 Knaus WA, Draper EA, Wagner DP and Zimmerman JE: APACHE II: a severity of disease classification system. Crit Care Med 13(10): 818-829, 1985. PMID: 3928249.

11 Vincent JL, Moreno R, Takala J, Willatts S, De Mendonça A, Bruining H, Reinhart CK, Suter PM and Thijs LG: The SOFA (Sepsis-related Organ Failure Assessment) score to describe organ dysfunction/failure. On behalf of the Working Group on Sepsis-Related Problems of the European Society of Intensive Care Medicine. Intensive Care Med 22(7): 707-710, 1996. PMID: 8844239. DOI: 10.1007/BF01709751

12 Vasileiadis I, Politou M, Dimopoulos S, Rovina N, Kyriakopoulou M, Kyriakoudi A, Tripodaki ES, Koutsouri T, Terpos E, Koulouris $\mathrm{N}$ and Koutsoukou A: Variation of endothelium-related hemostatic factors during sepsis. Microcirculation 25(8): e12500, 2018. PMID: 30159948. DOI: $10.1111 / \mathrm{micc} .12500$

13 Wang Y, Chen J, Ling M, López JA, Chung DW and Fu X: Hypochlorous acid generated by neutrophils inactivates ADAMTS13: an oxidative mechanism for regulating ADAMTS13 proteolytic activity during inflammation. J Biol Chem 290(3): 14221431, 2015. PMID: 25422322. DOI: 10.1074/jbc.M114.599084

14 Klebanoff SJ: Myeloperoxidase: friend and foe. J Leukoc Biol 77(5): 598-625, 2005. PMID: 15689384. DOI: 10.1189/jlb.1204697
15 Yende S, D'Angelo G, Kellum JA, Weissfeld L, Fine J, Welch RD, Kong L, Carter M, Angus DC and GenIMS Investigators: Inflammatory markers at hospital discharge predict subsequent mortality after pneumonia and sepsis. Am J Respir Crit Care Med 177(11): 1242-1247, 2008. PMID: 18369199. DOI: 10.1164/rccm.200712-1777OC

16 Arnhold J: The dual role of myeloperoxidase in immune response. Int J Mol Sci 21(21): 8057, 2020. PMID: 33137905. DOI: $10.3390 / \mathrm{ijms} 21218057$

17 Fukushima H, Nishio K, Asai H, Watanabe T, Seki T, Matsui H, Sugimoto M, Matsumoto M, Fujimura Y and Okuchi K: Ratio of von Willebrand factor propeptide to ADAMTS13 is associated with severity of sepsis. Shock 39(5): 409-414, 2013. PMID: 23481506. DOI: 10.1097/SHK.0b013e3182908ea7

18 Terpos E, Ntanasis-Stathopoulos I, Elalamy I, Kastritis E, Sergentanis TN, Politou M, Psaltopoulou T, Gerotziafas G and Dimopoulos MA: Hematological findings and complications of COVID-19. Am J Hematol 95(7): 834-847, 2020. PMID: 32282949. DOI: 10.1002/ajh.25829

19 Leclerc J, Pu Q, Corseaux D, Haddad E, Decoene C, Bordet R, Six I, Jude B and Vallet B: A single endotoxin injection in the rabbit causes prolonged blood vessel dysfunction and a procoagulant state. Crit Care Med 28(11): 3672-3678, 2000. PMID: 11098972. DOI: 10.1097/00003246-200011000-00023

20 Taylor FB, Haddad PA, Hack E, Chang AC, Peer GT, Morrissey JH, Li A, Allen RC, Wada H and Kinasewitz GT: Two-stage response to endotoxin infusion into normal human subjects: Correlation of blood phagocyte luminescence with clinical and laboratory markers of the inflammatory, hemostatic response. Crit Care Med 29(2): 326-334, 2001. PMID: 11269245. DOI: 10.1097/00003246-200102000-00020

Received December 31, 2021

Revised January 21, 2022

Accepted February 15, 2022 